conversations' from the outset and because they lacked coherent leadership. Interdisciplinary work requires particular skills, mindsets and attention to establishing common ground ${ }^{6-8}$.

\section{FACT FINDING}

Interdisciplinarity will be a headline topic at the GRC annual meeting in Delhi in May 2016, organized by India’s Science and Engineering Research Board and RCUK. A report on the state of play worldwide is being commissioned by RCUK, on behalf of the GRC (the team to undertake the research will be appointed in October).

The report will survey current policy and practice among global research funders. What forms of support do they offer to interdisciplinary research? How and where is it done? What are its outputs and impacts? The survey will begin to establish base data on how interdisciplinarity can best be stimulated and managed, and look for good practice in this most precious and complex of research endeavours.

The GRC expects to issue a policy statement following this meeting, as it has done previously on topical areas. These documents focus and clarify attitudes on key subjects. They marshal data that can be used while national policies are established and international cooperation is developed. We need much better definitions of what kind of thing we are supporting when and if we support interdisciplinary research, and better intelligence about what works. -

Rick Rylance is chief executive of the Arts and Humanities Research Council, chair of Research Councils UK, and a member of the governing board of the Global Research Council.

e-mail:r.rylance@ahrc.ac.uk

1. Lakhani, K. R., Jeppesen, L. B., Lohse, P. A. \& Panetta, J. A. The Value of Openness in Scientific Problem Solving Harvard Business School Working Paper (2007).

2. Elsevier. A Review of the UK's Interdisciplinary Research Using a Citation-based Approach (HEFCE, 2015).

3. Department for Business, Innovation \& Skills. Triennial Review of the Research Councils (BIS, 2014).

4. Klein, J. T. in The Oxford Handbook of Interdisciplinarity (eds Frodeman, R. et al.) 15-30 (Oxford Univ. Press, 2010).

5. German Research Foundation. Interdisciplinary Review Processes: Structural Impact and Funding Success (DFG, 2013); available at http://go.nature.com/uyfxlp (in German).

6. Bruce, A., Lyall, C., Tait, J. \& Williams, R. Futures 36, 457-470 (2004).

7. McLeish, T. \& Strang, V. Leading Interdisciplinary Research: Transforming the Academic Landscape (Leadership Foundation for Higher Education, 2014).

8. Whitfield, J. Nature 451, 872-873 (2008).

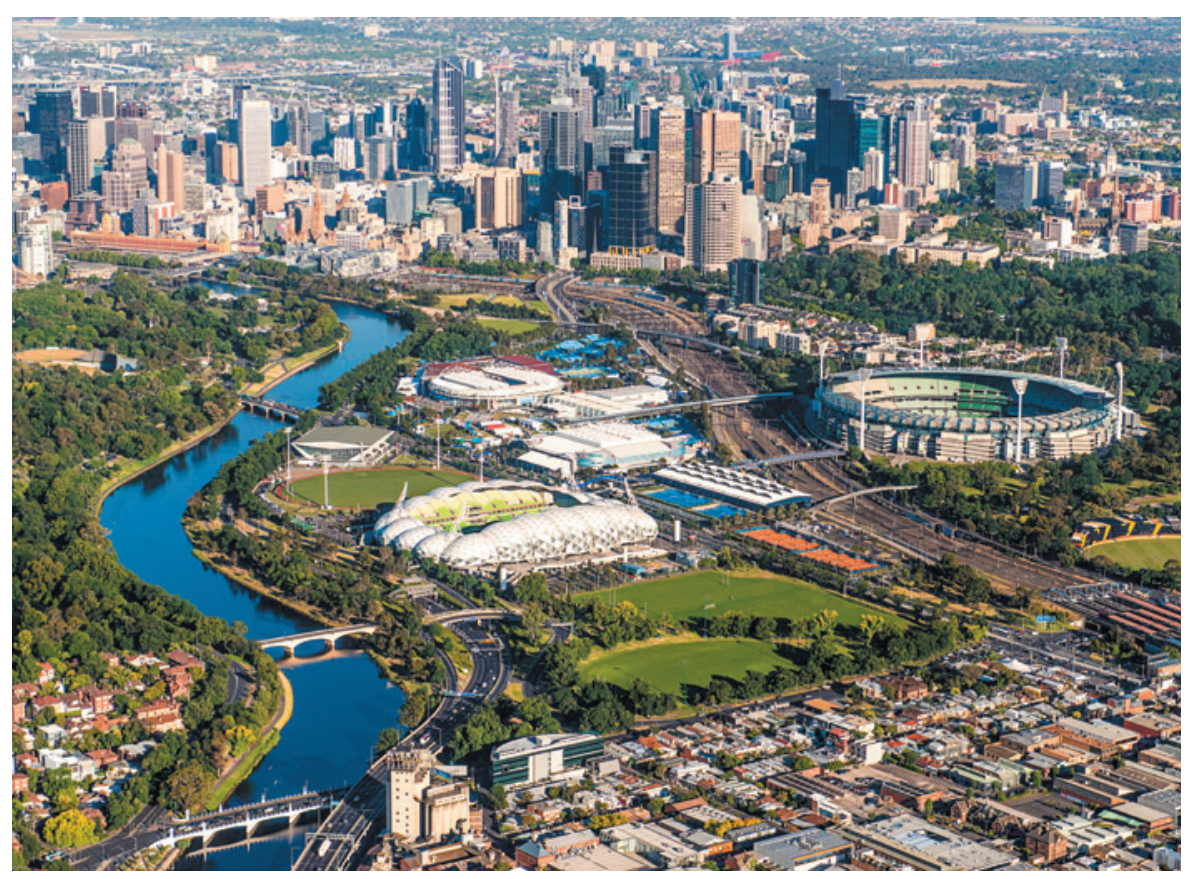

Equipping cities to weather our changing climate takes many disciplines working together.

\title{
How to catalyse collaboration
}

\section{Turn the fraught flirtation between the social and biophysical sciences into fruitful partnerships with these five principles, urge Rebekah R. Brown, Ana Deletic and Tony H. F. Wong.}

A $\mathrm{n}$ urgent push to bridge the divide between the biophysical and the social sciences is crucial. It is the only way to drive global sustainable development that delivers social inclusion, environmental sustainability and economic prosperity ${ }^{1}$. Sustainability is the classic 'wicked' problem², characterized by poorly defined requirements, unclear boundaries and contested causes that no single agency or discipline is able to address ${ }^{3}$.

It is crucial to understand, then, why so many well-meaning attempts at interdisciplinary collaboration fail to deliver tangible outcomes - and why others succeed. Here we offer an unapologetically personal answer by reflecting on how, working across multiple faculties of Monash University in Melbourne, Australia, we have built a team of

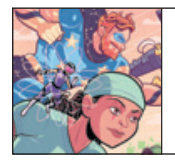
INTERDISCIPLINARITY A Nature special issue nature.com/inter disciplinary experts that delivers integrated and sustainable water management across multiple cities.

We have now grown this interdisciplinary team to incorporate other institutions nationally and internationally. At the same time, we acknowledge that substantial transaction costs come with interdisciplinary research it takes extra time and effort to make it work.

\section{PERSONAL JOURNEY}

Our journey began in the early 2000s, with two maturing groups working on urban water research: one in the faculty of engineering, focused on sustainable stormwater technologies, and the other in the faculty of arts, focused on urban water governance (see Supplementary Information; go.nature.com/ pjgbmn). The research teams had a common impact agenda, and our collaboration grew from a realization that an interdisciplinary approach would be more effective. In 2005, the two groups joined and secured funding for the establishment of a Aus\$4.5-million 
(US\$3.1-million) Facility for Advancing Water Biofiltration ${ }^{4}$ that brought together more than 20 Monash researchers and $\mathrm{PhD}$ students across civil engineering, ecology and sociology. By 2012, this had culminated in the award of a Aus\$120-million Cooperative Research Centre (CRC) for Water Sensitive Cities. It comprises a partnership of more than 85 organizations, including 13 research institutions, and around 230 researchers and $\mathrm{PhD}$ students from more than 20 disciplines and subdisciplines across the social and biophysical sciences and humanities.

Over the past decade, our collaborations have increasingly made a practical difference. We produce regular synthesis documents (see, for example, ref. 5) containing technology information and enabling policy advice, written in an accessible way to facilitate engagement and uptake. These have been heavily used in policy and strategy documents, which speeded up the adoption of our research. For example, stormwater regulations introduced in the state of Victoria in 2006 were underpinned by our research, and other state and local governments in Australia have adopted our recommended performance targets for the management of urban run-off. As a consequence, our stormwater-biofiltration technology has been increasingly adopted in cities across Australia $^{6}$, Singapore, China and Israel. Since 2010, our expanded framework for integrated city-wide water-cycle management ${ }^{7,8}$ has been used by governments (such as those of Australia, Singapore and China) and international organizations (such as the
Asian Development Bank) to guide their strategic planning and investment.

In that time, we have had to resolve considerable tension, which hinders meaningful collaboration. The biophysical sciences tend to have well-agreed theories; the social sciences spend much time developing (and often disagreeing on) theoretical questions. Both fields have control and comparison at their core. But biophysical researchers mainly perform quantitative research (often in well-controlled and replicable laboratory conditions), whereas social science can be qualitative or quantitative, and also use interpretative validation approaches.

We witnessed biophysical researchers accusing social scientists of poor rigour and of spending too much time conceptualizing problems without exploring and offering solutions. Conversely, social scientists were often frustrated that biophysical researchers were too focused on solutions, reductively overlooking the wider societal implications of their proposed solutions.

This discord is exacerbated by an inherent cultural hierarchy that often privileges the biophysical over the social sciences. Environmental problems have typically been framed from a biophysical perspective, meaning that social scientists are not effectively engaged in developing integrated solutions? .

\section{FIVE PRINCIPLES}

The journey was not for everyone, and we lost some talent along the way. Yet many stayed on. How did we help academics to overcome these biases? We used these five principles.
Forge a shared mission. Driving our collaborative journey was the shared mission of delivering water-management strategies that address the challenges of floods, droughts and degraded waterways. This approach fosters more sustainable, resilient, productive and liveable cities - for a healthy planet and population. The shared mission provided a compelling account of the overall goal of the collaboration, included impact as a necessary outcome, and was sufficiently broad to incorporate meaningful roles for all disciplinary researchers involved.

This mission also maintained a sense of purpose in the face of occasional failure and of the ongoing investment of huge time and effort to appreciate the norms, theories and approaches of other disciplines. When we needed the input of certain disciplines, and hastily included researchers that did not share the mission, it was not a success. The subsequent departure of these researchers from the team initially weakened the skill set of the group, but provided the motivation to expand our collaboration across multiple institutions.

Develop 'T-shaped' researchers. In our experience, interdisciplinary collaborations have the greatest chance of success when researchers are 'T-shaped ${ }^{10}$ - able to cultivate both their own discipline, and to look beyond it. Breadth and depth are key. T-shaped researchers build credibility by aiming for the highest scientific contribution in their field - a point of particular importance for early-career researchers, whose prospects

\section{MAKE IT MAINSTREAM}

\section{Ways to promote interdisciplinary research}

\section{Funders}

- Manage funding from an interdisciplinary perspective while reinforcing research impact. Discipline-based agencies must form joint funding programmes.

- Panels should include a balance of experts from the social and biophysical sciences, with a strong appreciation of other disciplines. It is also useful to include end-users of the research (for example, practioners and policymakers).

- Calls for funding should request balance between disciplines and prefer teams that have a proven record of collaboration. Publication in applicants' own disciplines should be essential; publishing in other disciplines is desirable.

\section{Institutions}

- Introduce key performance indicators that promote T-shaped researchers. For example, include qualitative measures of impact on policy and practice, as well as conventional academic indices. - Identify institutional research strengths that show potential for interdisciplinary collaboration and incentivize it through seed grants. - Reduce transaction costs: for example, through summer schools to develop constructive dialogue skills. Provide platforms - seminars, research workshops, debating competitions - to discuss challenges in cross-disciplinary research and offer insights into the norms and cultures of other disciplines. Co-locate researchers from different disciplines who work on the same grand challenges. - Invest in interdisciplinary PhD cohorts, co-supervised by academics from diverse departments or faculties.

\section{Publishers}

- Invest in and create high-quality interdisciplinary journals, managed by editorial teams or boards of T-shaped researchers. - Run special issues in high-impact, single-discipline journals that focus on interdisciplinary research.

- Peer reviewers should assess work using their disciplinary expertise, while being tasked to be open to innovations across disciplines.

\section{Researchers}

- Build stamina, patience and self-awareness to manage the long journey of establishing a productive interdisciplinary team.

- Put your best ideas forward even if they are unfinished, and be open to alternative perspectives from other disciplines, policymakers, industry practitioners and community members. - Prioritize depth early on, and embrace breadth by building relationships with those from other fields and practices. 
3. for promotion are judged against research 空 excellence criteria (see principle 5). T-shaped researchers also engage actively with other disciplines (see principle 3) to understand and appreciate their norms, theories, approaches and breakthroughs.

Many believe that interdisciplinary research delays career progression or is the luxury of senior researchers. This has not been our experience: many of our researchers were able to maintain a high publication rate in their own discipline, and - as part of a team - secure increasing interdisciplinary research funding. However, it took nearly five years to start publishing our joint interdisciplinary research in high-impact journals.

Nurture constructive dialogue. Through a decade of trial and error, we have invested heavily in creating the environment and informal rules that empower researchers across all sciences to engage effectively, despite their vastly different approaches to research design and methodology, and their differing technical vocabularies and communication cultures.

This has involved some commitments: to interact in plain English (disciplinary jargon is frowned on); to foster empathy and respect for different disciplinary norms; and to reflect on what is working in collaborative interactions. We designed regular interdisciplinary forums using these rules. This led to the codevelopment of key publications - for example, through interdisciplinary workshops, we have jointly written three annual reports for policymakers and water practitioners ${ }^{5}$. These activities grew into a sought-after annual short course and a massive open online course (MOOC) showcasing different disciplinary approaches to urban water challenges.

Reaching the ideal of constructive communication across the sciences takes time and practice - researchers new to the group may not yet have the necessary skills. Typically, they pass through three stages of development (see 'Journey to T'). Initially, new collaborators tend to dominate discussions and assert the primacy of their discipline. Soon after, they recognize the importance of other disciplines and adopt a more passive demeanour. Eventually, the researchers settle into a space of constructive dialogue.

We find that some quit and others stay to become mature collaborators, able to co-create across academic disciplines and broader networks. The role of more experienced collaborators is to support new colleagues' personal journeys into these dynamic relationships.

Give institutional support. Academic career pathways for interdisciplinary research are essential if it is to attract and

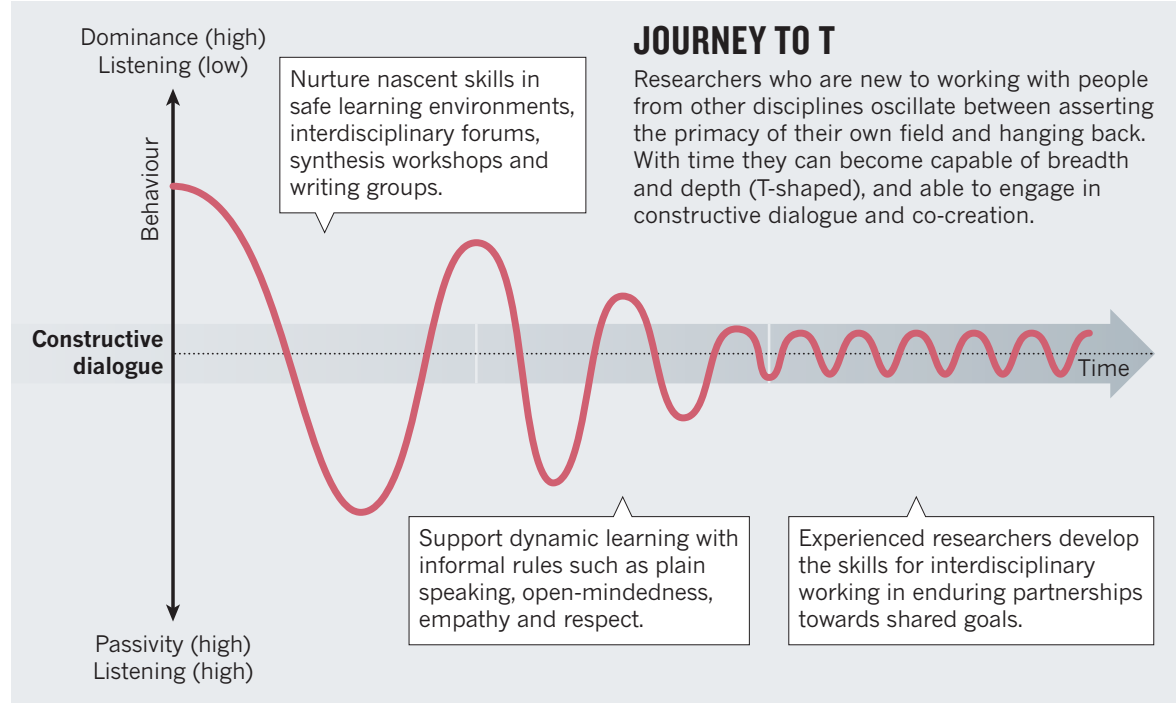

retain the brightest and best. Monash University's senior leadership team consistently signalled that it values research that is interdisciplinary, attracts significant industry involvement and delivers realworld impact - despite the organizational structures and global academic norms that are biased towards more conventional, disciplinary approaches.

This value was communicated to researchers through university policies, promotion criteria and seed-funding programmes. For example, the engineering faculty has introduced qualitative research standards (alongside the conventional quantitative measures), that

"Despite our
rewarding
experience,
interdisciplinary
research is
still on the
margins."

ties to catalyse collaborations.

Monash has established a $\mathrm{PhD}$ programme for cohorts of students working on a common global challenge across a number of disciplines; for instance, sustainable urban water management in developing Asian cities. These groups work in a constructive dialogue environment.

Bridge research, policy and practice. Finally, the establishment of enduring connections between researchers, policymakers and industry practitioners proved to be an important driver in growing our interdisciplinary collaborations. Refreshingly, industry rarely thinks in disciplinary silos. They tend to tackle complex problems from a range of perspectives, thereby modelling integrated, solution-focused thinking.

To ensure real-world impact, we engaged policy and industry partners in the design of our research programme and encouraged them to critique our scientific approach and presentation of results. We also ran frequent events that allowed professionals from policy and industry to interact with researchers. For example, in 2008, through a national roadshow, we showcased how our research is addressing crucial water challenges around Australian cities. Aimed at policymakers and industry and community leaders, it stimulated research and partnerships.

Despite our rewarding experience, interdisciplinary research is still on the margins. We urge researchers, institutions, and funding bodies committed to sustainable development to make it mainstream (see 'Ways to promote interdisciplinary research').

Rebekah R. Brown, Ana Deletic and Tony H. F. Wong are at Monash University in Melbourne, Australia, and in the Cooperative Research Centre for Water Sensitive Cities. R.R.B. is also director of the Monash Sustainability Institute. e-mail:rebekah.brown@monash.edu

1. United Nations. Transforming our World: The 2030 Agenda for Sustainable Development (UN, 2015).

2. Rittel, H. W. J. \& Weber, M. M. Policy Sci. 4, 155-169 (1973).

3. APSC. Tackling Wicked Problems: A Public Policy Perspective (Australian Government, 2007).

4. Deletic, A., Fletcher, T. D., Brown, R. R., Hatt, B. E. \& Wong, T. H. F. Water 35, 64-72 (2008).

5. Wong, T. H. F. et al. blueprint2013 - Stormwater Management in a Water Sensitive City (Cooperative Research Centre for Water Sensitive Cities, 2013).

6. Brown, R. R., Farrelly, M. A. \& Loorbach, D. A. Glob. Environ. Change 23, 701-718 (2013).

7. Wong T. H. F. \& Brown R. R. Water Sci. Technol. 60 , 673-682 (2009)

8. Brown, R. R., Keath, N. \& Wong, T. H. F. Water Sci. Technol. 59, 847-855 (2009).

9. ICSU. Earth System Science for Global Sustainability: The Grand Challenges (International Council for Science, 2010).

10. Hansen, M. \& von Oetinger, B. Harvard Bus. Rev. 79, 106-116 (2001). 